\title{
Die Anwendung der Finite-Elemente-Methode in der Lehre und Erzeugnisentwicklung an der Technischen Fachhochschule Wildau
}

\author{
Prof. Dr.-Ing. Peter Selke
}

\section{Einleitung}

Die Finite-Elemente-Methode (FEM) basiert in der heutigen Form auf der Matrizentheorie der Strukturmechanik, wie sie von ARGYRIS Ende der vierziger Jahre am Imperial College London und unabhängig davon von TURNER und CLOUGH bei Boeing in Seattle in Form der Matrizenkraftmethode für die Berechnung komplizierter Flugzeugrümpfe und Matrizenverschiebungsmethode für die Analyse der damals neuen Pfeilfliigel formuliert wurde. Sie stellt heute fuir den Ingenieur in Konstruktions-, Entwicklungs- und Forschungsabteilungen aller Branchen ein wichtiges Werkzeug für technische Berechnungen dar. Diese „Wunderwaffe der modernen Strukturmechanik"[1], wird inzwischen umfassend neben der Luft- und Raumfahrt und dem Maschinenbau in der Medizintechnik, der Elektrotechnik bis hin zur Konsumgiiterindustrie eingesetzt.

Für eine zeitgemäße, die technischen Entwicklungen beriicksichtigende Lehre ist daher die Einbeziehung der FEM in den Studienprozeß selbstverständlich. Dem trägt der Studienplan im Fachbereich Maschinenbau mit vier Semesterwochenstunden im 7. Semester in der Fachrichtung Konstruktion Rechnung.

\section{Lehrinhalte}

Die Methode der finiten Elemente entspricht im methodischen Vorgehen sehr der Denk- und Arbeitsweise des Ingenieurs. Sie vereinfacht die Modellfindung, wobei vielfach die Übereinstimmung mit dem realen Objekt verbessert werden kann.

Die Formulierung der zu lösenden Aufgabenstellung als Variationsproblem oder als Beziehung des gewichteten Restes, die Diskretisierung dieser Formulierung mit finiten Elementen und die Lösung des sich daraus ergebenden Gleichungspaketes mit digitalen Rechenanlagen mit der - in ihrer Bedeutung hervorzuhebenden - Verifikation der gewonnenen Rechenergebnisse stellen sich als grundlegende Verfahrensschritte fuir jedes Problem dar, bei dem der Anwender durch die Modellbildung, Vernetzungsstrategie und nicht zuletzt durch die Elementeauswahl großen Einfluß auf die Genauigkeit der Ergebnisse und die Effizienz der Berechnungen nimmt. Dies erfordert viel Erfahrung im Umgang mit den zumeist sehr allgemeinen, zunehmend komplexer werdenden Berechnungsprogrammen. Diese Erfahrung läßt sich allerdings nur in begrenztem Maße durch das Studium gewinnen. Praktische Anwendungen in Form von Di- plom- und Studienarbeiten fördern diesen Prozeß nachhaltig. Wenn man die FEM als moderne Computermechanik vermitteln und sie nicht auf reine Softwareschulung reduzieren will, dann muß bei vorausgesetztem mechanischen und mathematischen Grundwissen der Darstellung der Grundlagen ein ausreichender Zeitrahmen eingeräumt werden. Diesem Gedanken trägt der theoretische Teil der Lehrangebotes mit etwa $40 \%$ des zur Verfügung stehenden Zeitfonds Rechnung:

Bei der Abwägung zwischen der Erörterung einer Vielzahl von Anwendungen (z. B. der Hydro- und Gasdynamik, der elektromagnetischen Felder u. ä.) und dem tieferen Eingehen auf die speziellen Probleme der Festkörpermechanik wird im Maschinenbau unter Berüicksichtigung des Zeitfonds dem zweiten Gesichtspunkt Rechnung getragen. Neben der Darstellung der Grundlagen, die das erforderliche Wissen über den prinzipiellen Aufbau einer FEM-Rechnung vermitteln sollen, dem allgemeinen Aufbau eines Rechenprogramms, der Vorgehensweise der Verschiebungsmethode sowie auch Übungen zur Matrix-Steifigkeitsmethode ist das Verständnis der Arbeitssätze, hier das Prinzip der virtuellen Verschiebung unter Voraussetzungen der Grundgleichungen der linearen Elastizitätstheorie, Ziel und Gegenstand der Lehre. Die Formulierung des Dreieckelementes mit linearem Verschiebungsansatz (CST), Hinweise zu den Ansatzfunktionen und ein Überblick über den Elementekatalog für elastostatische Probleme beschließen den ,theoretischen" Teil.

Fuir den Erfolg der FEM in den letzten vierzig Jahren ist in erster Linie die Einheit von Theorie und Praxis, die sich als Folge der revolutionären Entwicklung der Rechentechnik eingestellt hat, zu sehen. Mit dem iiber dreißig Jahre alten Satz ,the computer shapes the theorie"[2], wird dieser Zusammenhang treffend beschrieben. Dies zu beruicksichtigen, bedeutet, einen wesentlichen Anteil des Studienangebotes für die Arbeit am Computer einzuräumen: Hierfür stehen an der Technischen Fachhochschule Wildau gegenwärtig die Programme „THOGASOFT“|6], „ANTRAS“|7| (beide auf PC 486/33) sowie das Programmpaket COSMOS/M|8] auf der Workstation IBM RS 6000 zur Verfuigung.

\section{Beiträge zur Erzeugnisentwicklung in der Wirtschaft}

Die im folgenden vorgestellten Beispiele fuir die FEM-Anwendung sind bis auf [5] aufgrund des Umfanges nicht im Rahmen des normalen Übungsbetriebes zu realisieren. 
Sie sind als Diplomarbeiten bzw. als Projektarbeiten nach Abschluß der FEM-Ausbildung füir die Mercedes-Benz AG Nutzfahrzeuge Ludwigsfelde GmbH, die EUROCON GmbH Berlin sowie die Institut fuir Schienenfahrzeuge GmbH Berlin entstanden.

\section{Beispiel: Gestaltung und Optimierung eines Motorträgers [3]}

Untersuchung von vier Varianten bei Annahme von sechs Lastfällen mit dem Ziel den Fertigungsaufwand, die Masse sowie die Spannungen und Verformungen zu minimieren.

Unternehmen: Mercedes-Benz AG Nutzfahrzeuge Ludwigsfelde GmbH

Rechenmodell: numerische Rechnung mit FEM-Programm ANTRAS auf PC 486/33; Bauteilvernetzung: 878...1900 Volumen-Viereck-Elemente, 1389 ...2957 Knoten Ergebnisse: Bilder $1 \ldots 4$

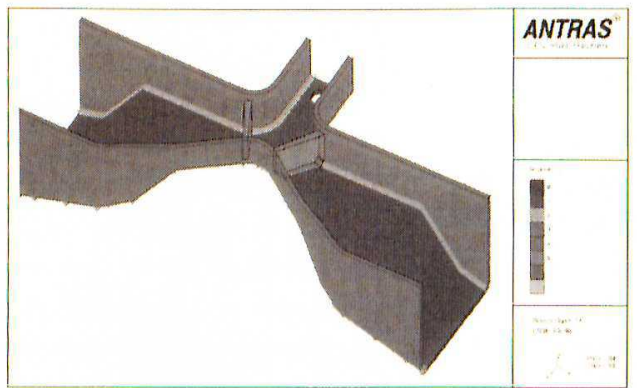

Bild 1: Modellierung des Motortrügers

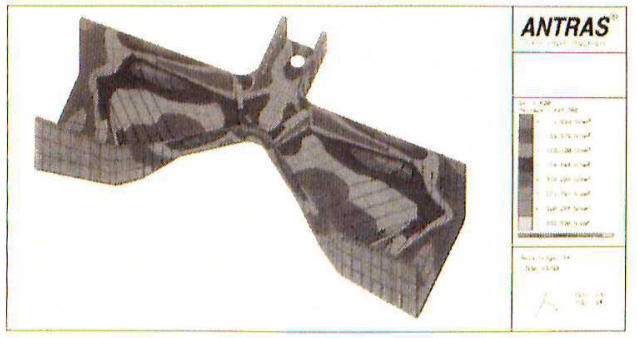

Bild 2: Spannungsverteilung (Vergleichsspannungen) des Motorträgers

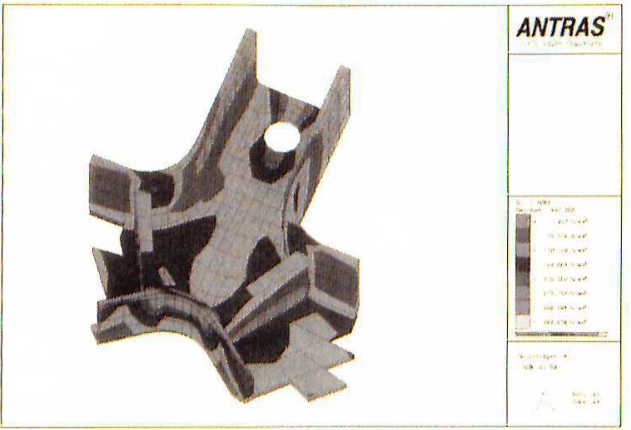

Bild 3: Verteilung der Vergleichsspannungen (Ausschnitt)

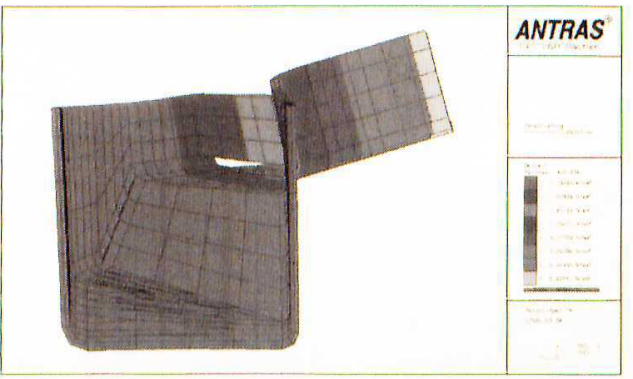

Bild 4: Verfornung des Motorträgers
2. Beispiel: Optimierung eines Lenkgetriebeblockes [4] Untersuchung von vier Varianten hinsichtlich des Abbaus von Spannungsspitzen an kritischen Stellen und Begrenzungen der Verformung bei Reduzierung der Masse für drei Lastfälle.

Unternehmen: Mercedes-Benz AG Nutzfahrzeuge Ludwigsfelde $\mathrm{GmbH}$

Rechenmodell: numerische Rechnung mit FEM-Programm COSMOS/M auf Workstation IBM RS 6000; Bauteilvernetzung: 4376 ...7026 Volumenelemente SOLID, 6483... 14343 Knoten

Ergebnisse: Bilder 5...9

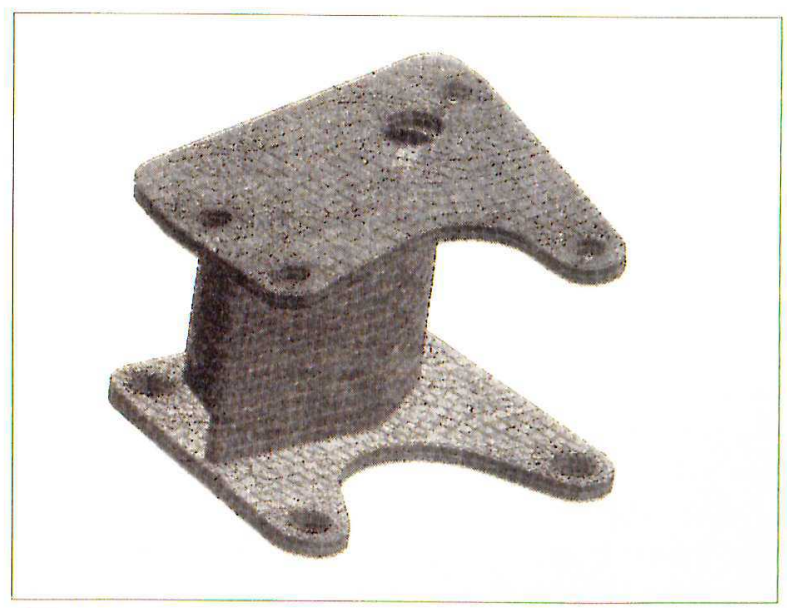

Bild 5: Mit SOLID-Elementen vernetzter Lenkgetriebeblock

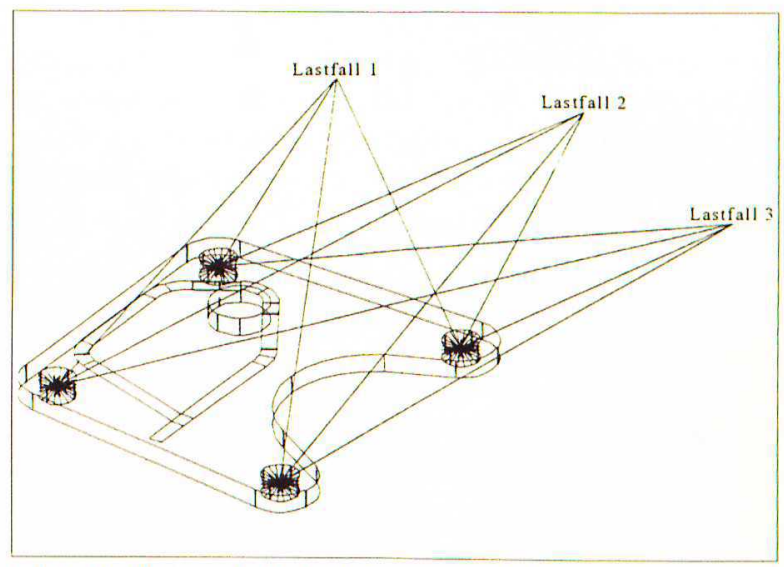

Bild 6: Modellierung der Krafteinleitung an den Bohrungen mit starren Verbindungselementen

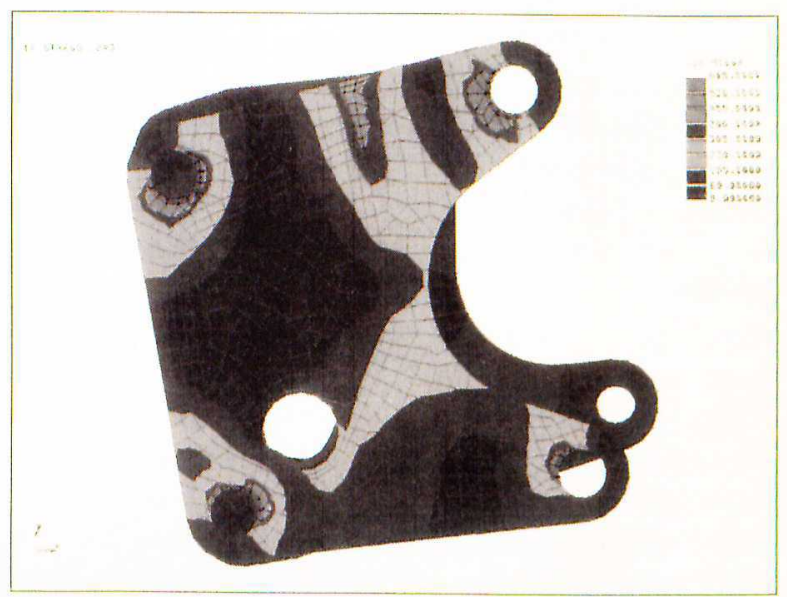

Bild 7: Vergleichsspannung Basismodell (Ansicht von unten) 


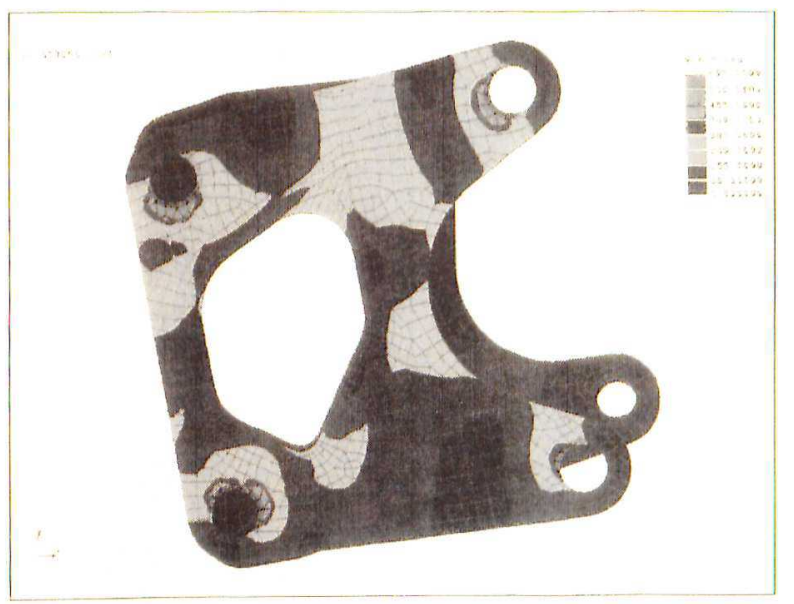

Bild 8: Vergleichsspannung Variante I (Ansicht von unten)

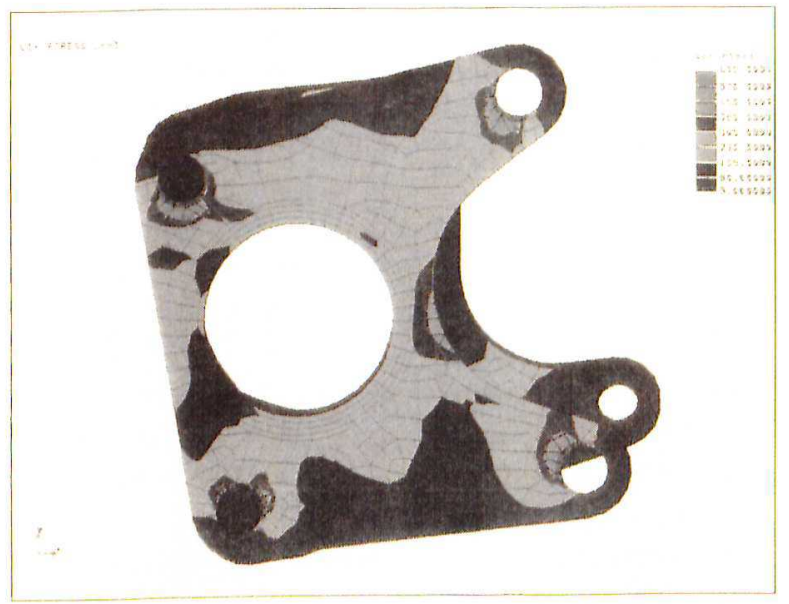

Bild 9: Vergleichsspannung Variante 2 (Ansicht von unten)

\section{Beispiel: Verformungsberechnung eines Containerbodens} Berechnung der elastischen Biegeverformung und der Vergleichsspannung der Bodengruppe eines Containers für den Gefahrguttransport bei einer konstant verteilten Flächenlast für zwei Betriebszustände.

Unternehmen: EUROCON GmbH Berlin

Rechenmodell: numerische Rechnung mit FEM-Programm COSMOS/M auf Workstation IBM RS 6000; Bauteilvernetzung: 2408 Schalenelemente SHELL 4, 1169 Balkenelemente BEAM 3D, 4235 Knoten Ergebnisse: Bild 10

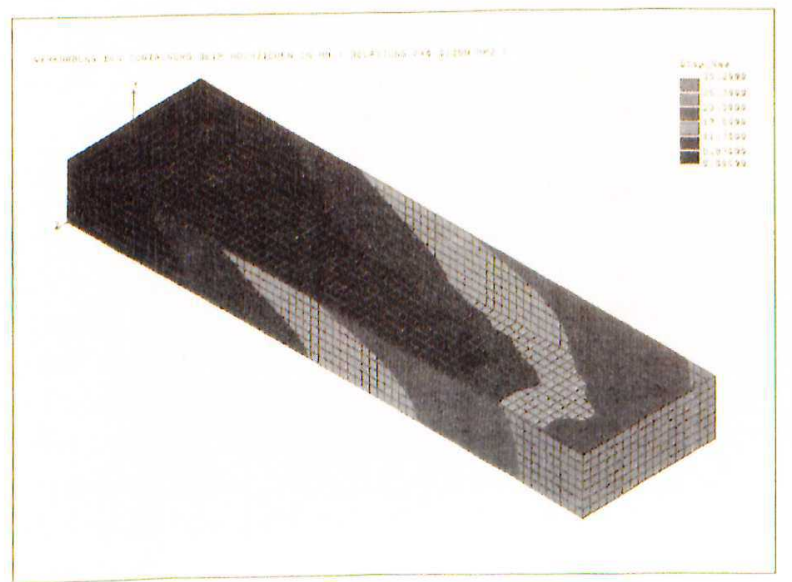

Bild 10: Verformung des Containers beim Hochziehen
4. Beispiel: Spannungsberechnung einer Scheibe [5]

Berechnung der elastischen Biegeverformung und der Maximalspannung sowie Analyse der Spannungsverteilung der Außenscheibe eines Hochgeschwindigkeitszuges bei vorgegebener Flächenlast (Extremwert der Luftdruckschwankung nach Anforderungsliste).

Untemehmen: Institut für Schienenfahrzeuge GmbH Berlin Rechenmodell: numerische Rechnung mit FEM-Programm COSMOS/M auf Workstation IBM RS 6000; Bauteilvernetzung: 4800 Schalenelemente SHELL4, 4941 Knoten Ergebnisse: Bild 11

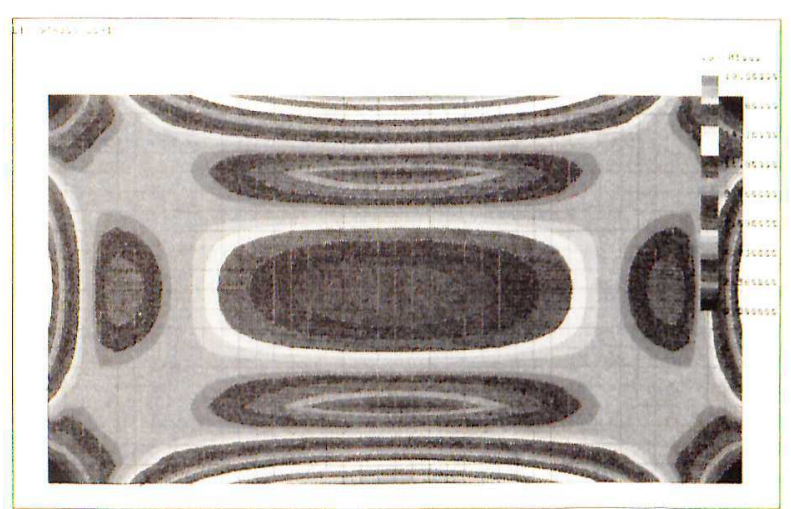

Bild 11: Vergleichsspannungen der Außenscheibe

Das letzte Beispiel, die am gesamten Umfang eingespannte Platte (Bild 11), zeigt sehr anschaulich, daß der Einsatz der FEM nicht nur bei der Berechnung komplizierter Bauteilformen sinnvoll sein kann, sondern sich auch mit geringem Eingabeaufwand für einen vorgebildeten Nutzer (Abschluß des Lehrgebietes) gute Ergebnisse, die auf analytischem Wege eher muihevoll zu gewinnen sind, sehr schnell erzielen lassen.

Der Autor dankt an dieser Stelle ausdrücklich den hier genannten Unternehmen für die freundliche Genehmigung der Darstellung der Ergebnisse.

\section{Literatur}

11| MATTHECK, C.: „Design in der Natur“, Freiburg: Rombach Verlag 1992, S. 27

12| ARGYRIS, J., MLEJNEK, H.-P. : „Die Methode der Finiten Elemente“ Band I, S. XII,Braunschweig: Friedrich Vieweg \& Sohn 1994

13| LIEBL, T.: „Auslegung des hinteren Motorträgers für den NKW MB I.K mit 4-Zylinder-Motor und Getriebe", Diplomarbeit, TFH Wildau 1994

14| PRÜFER, R.: „Optimierung eines Lenkgetriebeblockes mittels FEM“* Diplomarbeit, TFH Wildau 1994

515 SCHUBERT, K.: „Projektierung eines Fensters für den Hochgeschwindigkeitszug im Zusammenhang mit der aktiven Isolierung". Diplomarbeit, TFH Wildau 1994

|6| „THOGASOFT", TGS Software-Verlag-Hardware, Wallenhorst

17| "ANTRAS", Gesellschaft für Strukturanalyse GfS Aachen

|81 „COSMOS/M“, SRAC Santa Monica/USA

\section{Verfasser}

Prof. Dr.-Ing. Peter Selke

Technische Fachhochschule Wildau

Fachbereich Maschinenbau

Tel. (03375) 507-115 